\title{
AN INTEGRATED MODELING APPROACH THAT COMBINES ELASTIC AMPLIFICATION AND ROCKING ANALYSIS FOR SEISMIC ASSESSMENT OF A MASONRY TOWER
}

\author{
ANJALI MEHROTRA ${ }^{1 *}$, ANDREW LIEW ${ }^{2}$, PHILIPPE BLOCK ${ }^{3}$ AND MATTHEW J. \\ DEJONG ${ }^{4}$ \\ ${ }^{1}$ Institute for Sustainability and Innovation in Structural Engineering \\ University of Minho, Portugal \\ e-mail: anjali.a.mehrotra@gmail.com (*corresponding author) \\ 2 Department of Civil and Structural Engineering \\ University of Sheffield, UK \\ email: a.liew@sheffield.ac.uk \\ ${ }^{3}$ Block Research Group - Institute for Technology in Architecture \\ ETH Zurich, Switzerland \\ email: block@arch.ethz.ch \\ ${ }^{4}$ Department of Civil and Environmental Engineering \\ University of California - Berkeley, USA \\ e-mail: dejong@berkeley.edu
}

Keywords: Masonry, Rocking Dynamics, Finite Element Modeling, Amplification Effects, Computational Tools

\begin{abstract}
Failure of masonry structures during earthquakes often occurs via specific, welldocumented collapse mechanisms, many of which involve partial collapse of the structure well above ground level. Consequently, the elastic response of the structure needs to be considered, which in the case of historic structures such as bell towers and churches often requires modal analysis using finite element models - the generation of which can be labour-intensive and timeconsuming. This paper presents a new integrated modeling approach which combines finite element analysis with rocking dynamics in order to model the seismic response of complex structural geometries in a computationally-efficient manner. The modeling strategy is implemented within COMPAS - an open-source computational framework that provides geometry processing independent of CAD software, and is incorporated within the broader framework of a tool being developed for the seismic collapse assessment of masonry structures. The framework of this new tool is first outlined, and the utility of the new modeling approach then demonstrated through application to the seismic assessment of a historic masonry tower in North-Eastern Italy. The analysis results indicate that for the level of seismic hazard expected on site, failure of the tower is most likely to occur via overturning collapse of one of the rampart elements. The importance of accounting for elastic amplification effects, as well as the influence of varying boundary conditions on the dynamic response, is also demonstrated.
\end{abstract}




\section{INTRODUCTION}

Masonry structures are susceptible to collapse under the influence of seismic action. When such structures fail, they often do so via specific, well-documented collapse mechanisms ([1], [2]) - analysis of which can be conducted using either simplified analytical procedures (as implemented in many building codes) or through the use of more complex numerical modeling strategies (such as finite element analyses or discrete element methods). While the former can be over-conservative, often underestimating dynamic capacity and leading to expensive - and at times unnecessary - retrofitting solutions, the latter can be fairly computationally-expensive and time-consuming, especially when trying to model collapse. As an alternative analysis approach, rocking dynamics, whereby equations of motion describing different collapse mechanisms are directly derived and solved, can be used instead ([3]-[6]). Such an approach has the advantage of being less computationally-demanding than most numerical models, while providing more accurate predictions than most simplified analytical methods.

However, assessment of real structures often requires consideration of numerous different collapse mechanisms, many of which involve partial collapse of the structure well above ground level. Thus amplification and filtering of the ground motion by the building - which depends in turn on the natural frequency of the structure, also needs to be taken into account [7]. While the natural frequency and modes of simple, regular structures can be determined analytically, for many historic structures (e.g. bell towers, churches) consideration of the elastic response requires modal analysis using finite element models ([8]-[10]), the generation of which can be

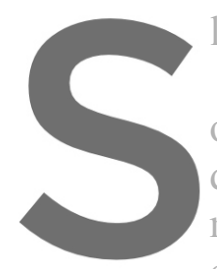
labour-intensive and tim

\section{In this paper, a new} of the structure as inpu dynamics to model the manner. The modelin

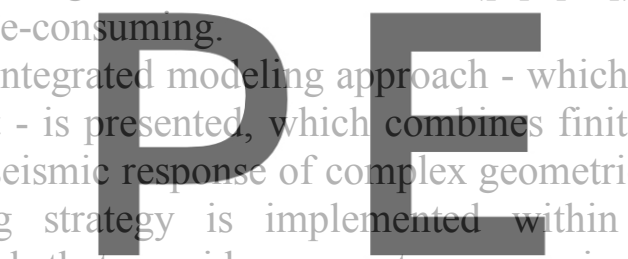
computational framework that provides geo

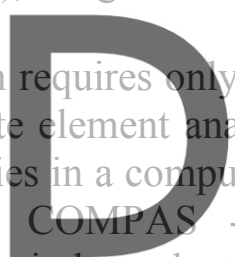

geometry processing

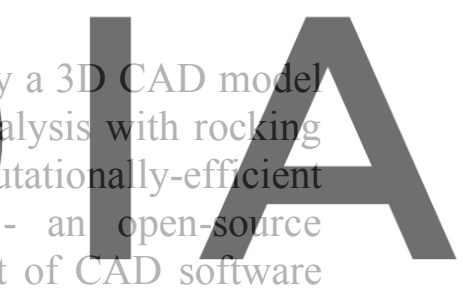
[11], and is incorporated within the broader framework of a tool being developed for the seismic

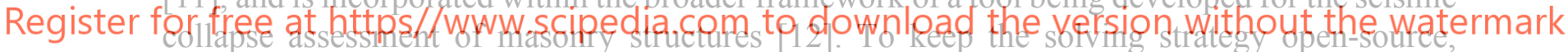
modal analyses are conducted using the compas fea package [13], which directly constructs the FE model from the input geometry, and analyses it using the open-source finite element solver OpenSees [14]. The results of the modal analysis are subsequently used to define an equivalent single-degree-of-freedom (SDF) oscillator, which is subjected to different ground motion records. The response of the oscillator is then scaled appropriately, with the scaled response serving as the input signal at the base of the rocking mechanism. The procedure can be repeated for a wide range of potential collapse mechanisms within a structure, to determine the most vulnerable mechanisms for a given suite of ground motions. The utility of this new modeling approach is finally demonstrated by applying it to the seismic assessment of a historic clock tower in Lendinara, Italy.

\section{METHODOLOGY}

A flowchart outlining the functioning of this proposed tool can be found in Figure 1. 


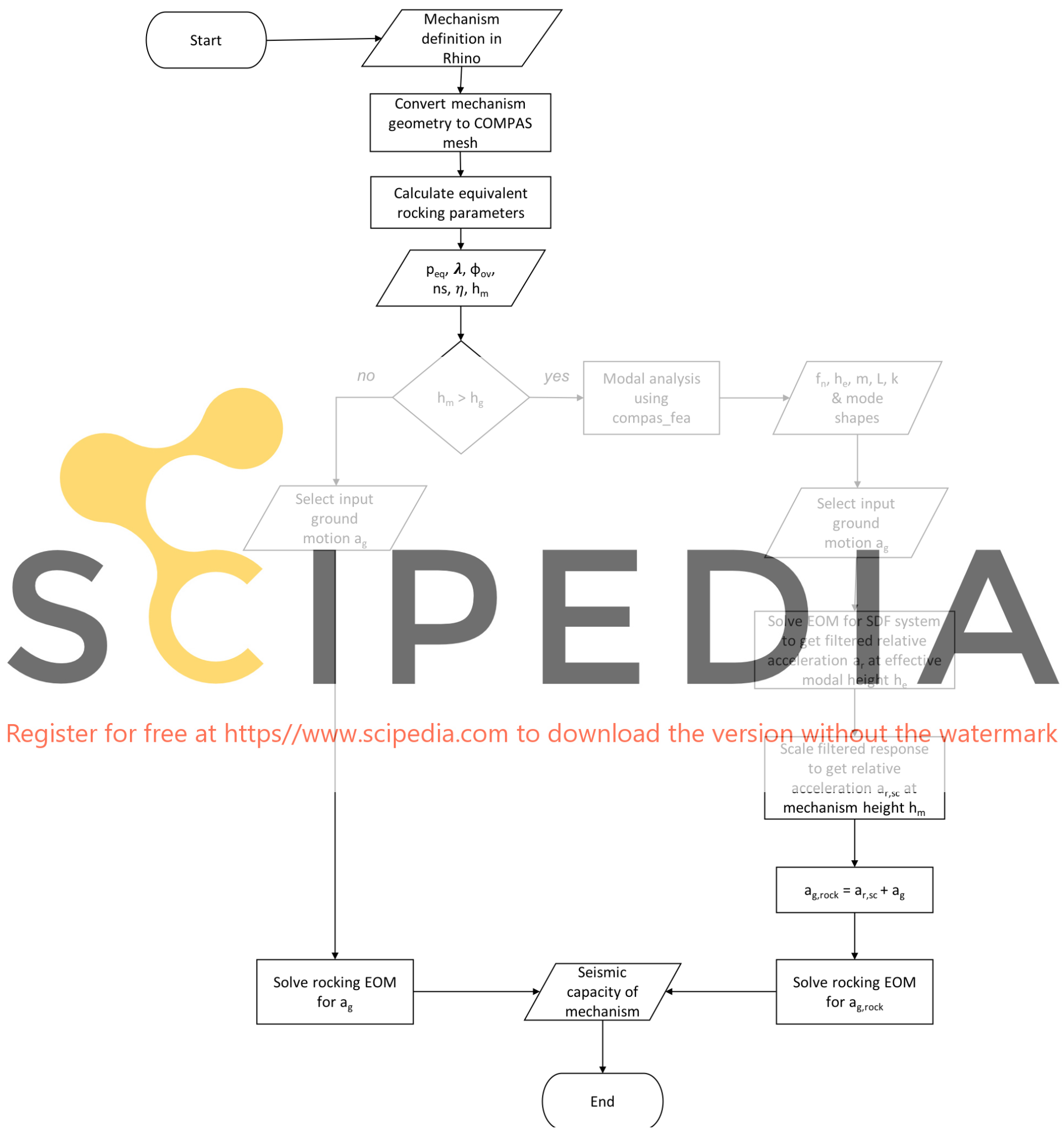

Figure 1: Flowchart illustrating the functioning of the proposed computational tool/modeling approach 


\subsection{Geometry and mechanism definition in the CAD interface}

As stated in the introduction, the proposed modeling strategy only requires a 3D CAD model of the structure as input. However, as this strategy is implemented within COMPAS, which is a Python-based framework, it is crucial that the CAD program used is capable of editing and executing Python-based scripts. To that end, the CAD program Rhino [15] was selected as the preliminary interface for this tool, as it not only comes with its own Python interpreter, but also works on both Windows and Mac computers.

Once the geometry of the whole structure has been defined in Rhino, the portion of the structure involved in the collapse mechanism needs to be demarcated. The mechanism geometry is then meshed in Rhino, and the compas_rhino.helpers package is subsequently used to convert this Rhino mesh into its COMPAS counterpart - essentially converting the 3D solid into a datastructure defined by a set of vertices and faces. The axis of rotation as defined by the user is also saved as an attribute of this mesh so that it can be easily accessed in the next stage of analysis. If the mechanism takes place above ground level, a second mesh comprising the entire structure is also created and converted into its COMPAS counterpart. Attention must be paid to the density of this second mesh as it serves as the basis of the finite element model used for the modal analysis.

However, as the mesh datastructures as exported from Rhino comprises only 2D face meshes, i.e. only the external faces of the solids are meshed, the meshes first need to be converted into 3D tetrahedral meshes (i.e. internally meshed) before any operations can be

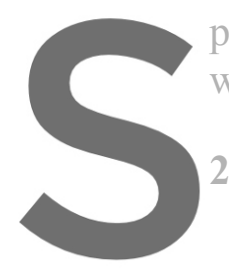
performed on it within the COMPAS framework. This is done
which in turn provides a Python interface to program TetGen
2.2 Calculation of equivalent rocking paranneters
Once the mechanism geometry has been converted to the 3

Once the mechanism geometry has been converted to the 3D mesh datastructure.

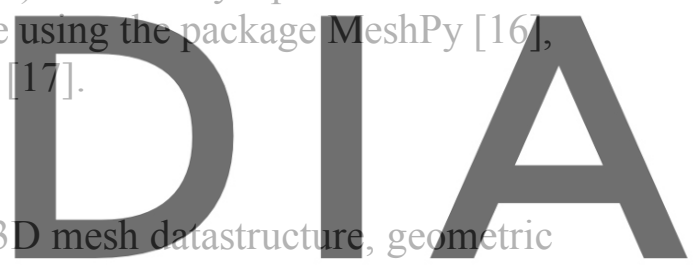

operations can be directly performed on it within the COMPAS environment. These include

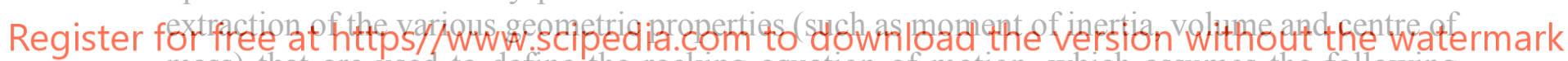
mass) that are used to define the rocking equation of motion, which assumes the following general linearized form [4]:

$$
\tilde{I} \ddot{\phi}-\tilde{K}\left(\phi-\phi_{c r}\right)=-\tilde{B} \ddot{u}_{g}+\tilde{M}
$$

where $\tilde{I}$, is the moment of inertia of collapsed portion of the structure about the axis of rotation, $\widetilde{M}$ is the moment caused by the external static forces, $\phi_{c r}$ is the critical rotation, $\widetilde{K}$ is the rotational stiffness of the system, and $\tilde{B} \ddot{u}_{g}$ is the moment provided by the ground motion applied to the structure. Note that the linearization here has occurred about the point of unstable equilibrium (i.e. $\phi=\phi_{c r}$ ) in order to obtain local dynamic equivalence with the single rocking block. This single equation of motion can then be used to describe a variety of different mechanisms, ranging from the overturning of a single rocking block to the dynamic behaviour of more complex two and three block mechanisms.

By using the following transformation of variables:

$$
\theta=\phi \frac{\tilde{K}}{g \tilde{B}}
$$

Equation (1) can be rewritten as: 


$$
\ddot{\theta}=p_{e q}^{2}\left(\theta-\lambda-\frac{\ddot{g}}{g}\right)
$$

where $p_{e q}$ is the rocking frequency parameter and $\lambda$ is an approximation of the static load multiplier that activates the mechanism. Both these terms depend on the kinematic constants $\tilde{I}$, $\widetilde{K}, \widetilde{B}, \widetilde{M}$ and $\phi_{c r}$, which in turn depend on both the geometry of the structure as well as the type of collapse mechanism; more detailed expressions for which can be found in [12].

Additionally, the overturning rotation $\phi_{o v}$, that is, the rotation upon the exceedance of which the structure will overturn and collapse, can also be calculated using the aforementioned kinematic constants as shown below:

$$
\phi_{o v}=\phi_{c r}-\frac{\widetilde{M}}{\widetilde{K}}
$$

Moreover, in the case of impact, energy dissipation by the block(s) is accounted for through the coefficient of restitution $\eta$, which depends not only on the geometry of the blocks but also on the type of rocking, i.e. one or two-sided - indicated in this paper by the term $n s$. For more detailed expressions for the coefficient of restitution, please refer to [12]. Finally, the height $h_{m}$ at which the mechanism occurs is determined by taking the average of the heights of the two points defining the axis of rotation, previously saved as an attribute of the mesh.

\subsection{Modal analysis using compas_fea}

In the case of mechanisms that take place above ground level (i.e. $h_{m}>h_{g}$ ) modal analyses

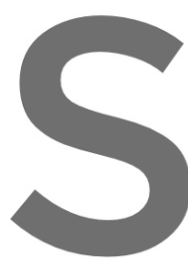
need to be conducted to define an in turn is used to account fo structure.

Using the tetrahedral package is utilized for the const
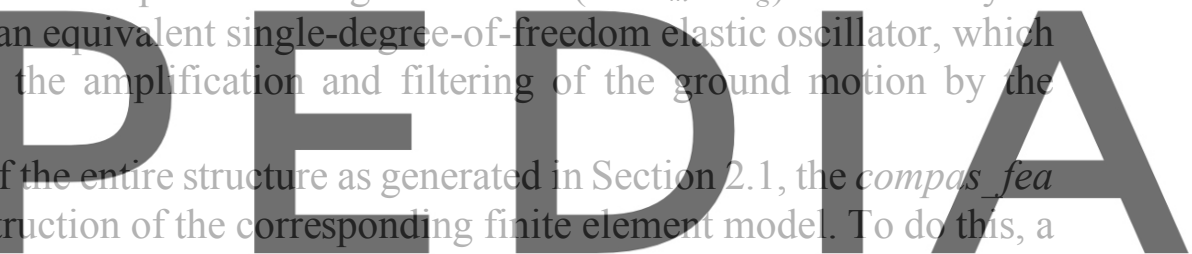

Structure object is first created, with nodes corresponding to each of the points of the tetrahedral

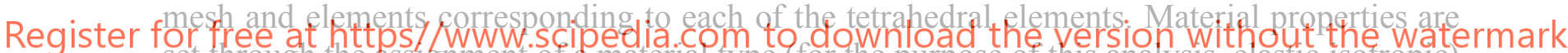
set through the assignment of a material type (for the purpose of this analysis, elastic isotropic) which in turn is characterized by a user-defined Young's modulus, Poisson's ratio and density. Boundary conditions are specified through the assignment of pinned displacements to predefined node sets, which are applied through the addition of a Step object. A second Step object, in this case ModalStep, is then added to instruct the finite element solver to conduct a modal analysis, specifying the number of modes to be analysed.

After the Structure object has been constructed, it is written to a .tcl input file which is subsequently sent to OpenSees for analysis. The analysis is conducted in the background and once completed, the results - such as modal frequencies $f_{n}$ and nodal displacements $u$ for each mode - are returned. Post-processing of this data results in the structure being transformed into an equivalent single-degree-of-freedom elastic oscillator, defined by the following equation of motion:

$$
\widetilde{m} \ddot{z}+2 \widetilde{m} \xi \omega_{n} \dot{z}+\tilde{k} z=-\tilde{L} \ddot{u_{g}}
$$

where:

$$
\widetilde{m}=\sum_{i=1}^{N} m_{i} u_{i}^{2} ; \quad \tilde{L}=\sum_{i=1}^{N} m_{i} u_{i} ; \quad \omega_{n}=2 \pi f_{n} \text { and } \tilde{k}=\widetilde{m} \omega_{n}^{2}
$$


where $m_{i}$ is the mass lumped into each of the nodes of the model and $u_{i}$ their corresponding displacements. System damping is specified through the term $\xi$, and is set to $5 \%$ for the analyses conducted in this paper. Solving Equation (5) gives the response of the oscillator at the effective modal height $h_{e}$, which is determined using the following expression:

$$
h_{e}=\frac{\sum_{i=1}^{N} m_{i} u_{i} h_{i}}{\sum_{i=1}^{N} m_{i} u_{i}}
$$

where $h_{i}$ is the height of each node. However, for mechanisms that occur at a height above or below $h_{e}$ (i.e. $h_{m} \neq h_{e}$ ), the response needs to be scaled using the mode shapes, which are extracted using the nodal displacements $u$. This is illustrated by Figure 2 for a sample tower with varying boundary conditions, including the isolated case, that is, only base nodes pinned and the "fixed" case, that is, nodes on either side of the structure also pinned up to a certain height, to simulate the connectivity of the tower to adjacent buildings.
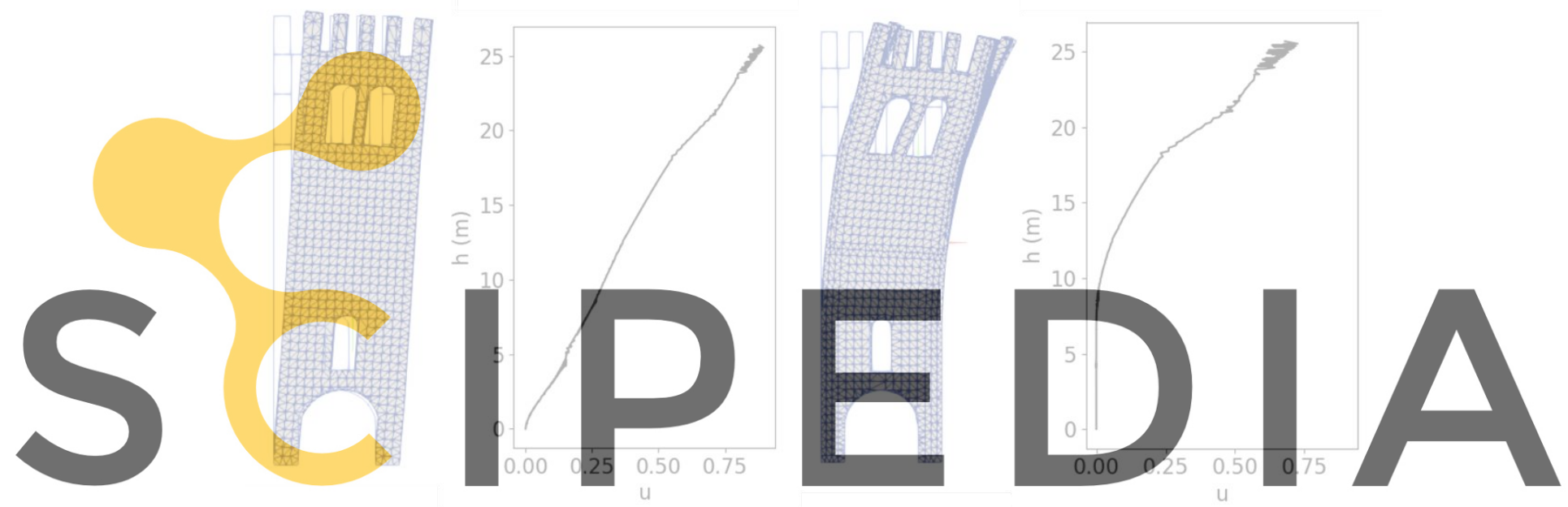

Register for free at httpsisphaturw.scipedia.com to download the version without the watermark

Figure 2: First mode shapes extracted by the tool for a sample tower, for both the isolated case as well as the case where the tower is connected to the adjacent buildings ("fixed" case)

\subsection{Full time-history analysis}

In the case of full time-history analyses, Equation (5) is first solved for the unscaled input ground acceleration $a_{g}$ (i.e. $\ddot{u}_{g}=a_{g}$, Figure $3 \mathrm{a}$ ). The solution to the equation of motion yields the filtered response of the structure $a_{r}$ - relative to the ground - at the effective modal height $h_{e}$ (Figure 3b). For mechanisms that occur at a height other than $h_{e}\left(h_{m} \neq h_{e}\right), a_{r}$ then needs to be scaled using the mode shapes (Figure 2), to get the filtered and scaled response $a_{r, s c}$ at the mechanism height $h_{m}$ (Figure 3c), as shown below:

$$
a_{r, s c}=\frac{u\left(h_{m}\right)}{u\left(h_{e}\right)} a_{r}
$$

However, $a_{r, s c}$ is only the scaled and filtered response of the structure relative to the ground. Thus to get the total acceleration experienced by the structure at the base of the rocking mechanism, this acceleration $a_{r, s c}$ is added to the original input ground acceleration $a_{g}$ and the final combined acceleration is illustrated by Figure $3 \mathrm{~d}$. This acceleration $a_{g \text {,rock }}$ serves as the 
final input signal for the rocking equation of motion (Equation (3), $\ddot{u}_{g}=a_{g, \text { rock }}$ ), which is then solved to predict the response of the rocking mechanism in terms of rotation $\phi$ over time.
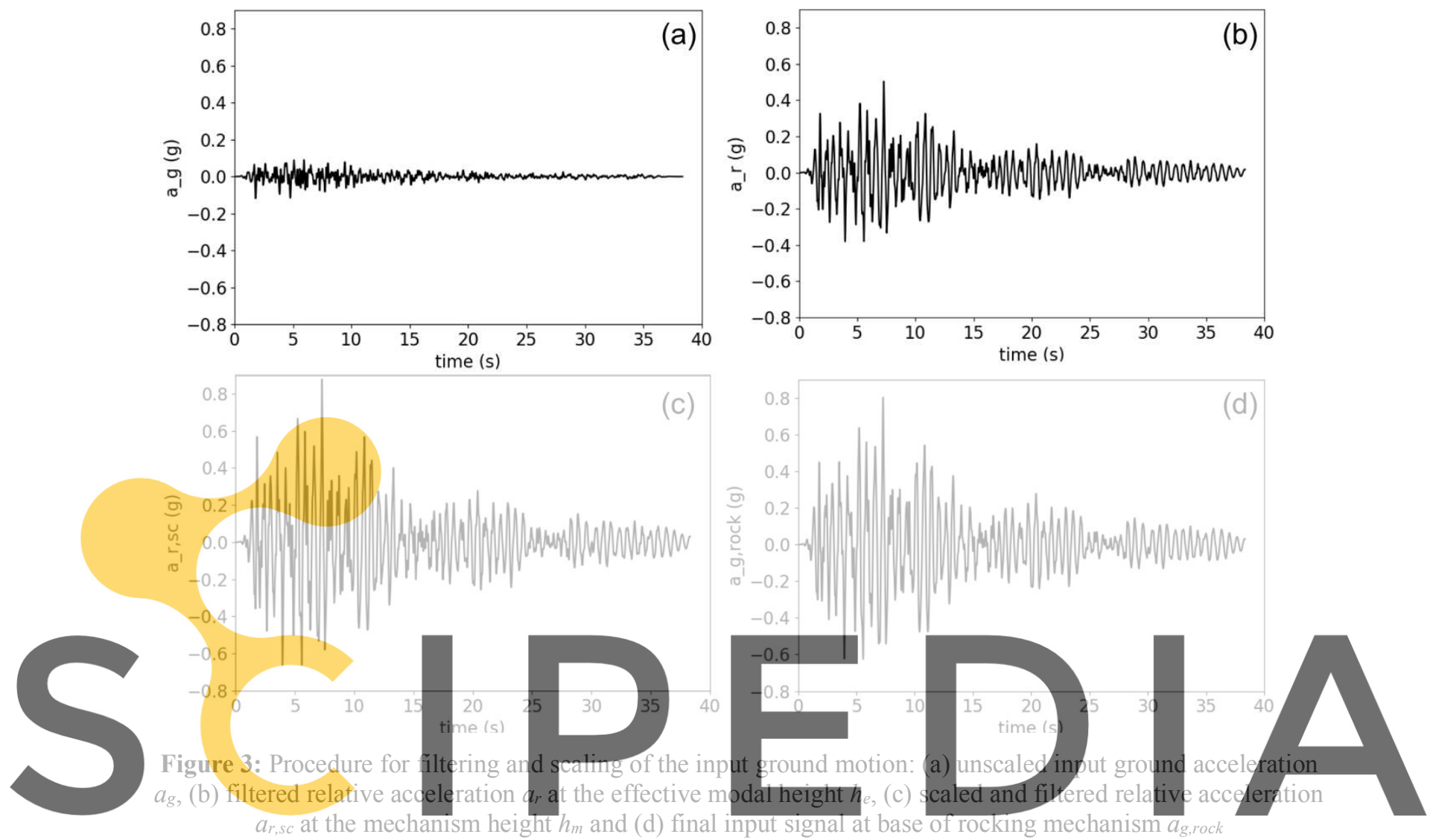

Register for fresatshtps// wworscipedia:વon todowndoad the version without the watermark

To demonstrate this new integrated modeling approach, a clock tower in the city of Lendinara, Italy was chosen as a case study. Full time-history analyses were conducted for a predefined set of collapse mechanisms, and the results compared to the expected seismic hazard in the region.

\subsection{Geometry of the tower and seismic hazard of the area}

The clock tower chosen for analysis is approximately square in plan and constructed entirely in brick, with a height of $25.7 \mathrm{~m}$ and base dimensions of $7.2 \times 8.0 \mathrm{~m}$ [18]. The walls are roughly $100 \mathrm{~cm}$ thick for the bottom $12.6 \mathrm{~m}$, with this thickness reducing to $50 \mathrm{~cm}$ near the top [18]. Two large arches are present on the eastern and western façades at the bottom of the structure, which enable the tower to connect the two small squares that it borders, while all four façades have large double openings at the upper level of the structure, with merlon (rampart) elements present at the very top [18].

The tower is located in North-Eastern Italy in a zone of low seismicity, with a maximum PGA of $0.075-0.100 \mathrm{~g}$ expected with a $10 \%$ probability of exceedance in 50 years. In this analysis, the ground motion applied to the tower will thus be scaled to PGA $=0.1 \mathrm{~g}$ (or some 
factor thereof) to gauge the vulnerability of the structure to collapse.

\subsection{Mechanisms selected for analysis}

As illustrated by Figure 4, three different collapse mechanisms were selected for analysis, all of which occur high enough up so that amplification effects will not be negligible. These include the overturning of one of the merlon/rampart elements at the very top of the tower (Mechanism a1), as well as corner failure with cracks originating at the large double openings. In the case of the corner mechanisms, the crack angles were chosen to represent both an average value $\left(\alpha_{c}=45^{\circ}\right.$, Mechanism b1) as well as an upper limit $\left(\alpha_{c}=70^{\circ}\right.$, Mechanism b2) for a range of different brick aspect ratios and bonding patterns.
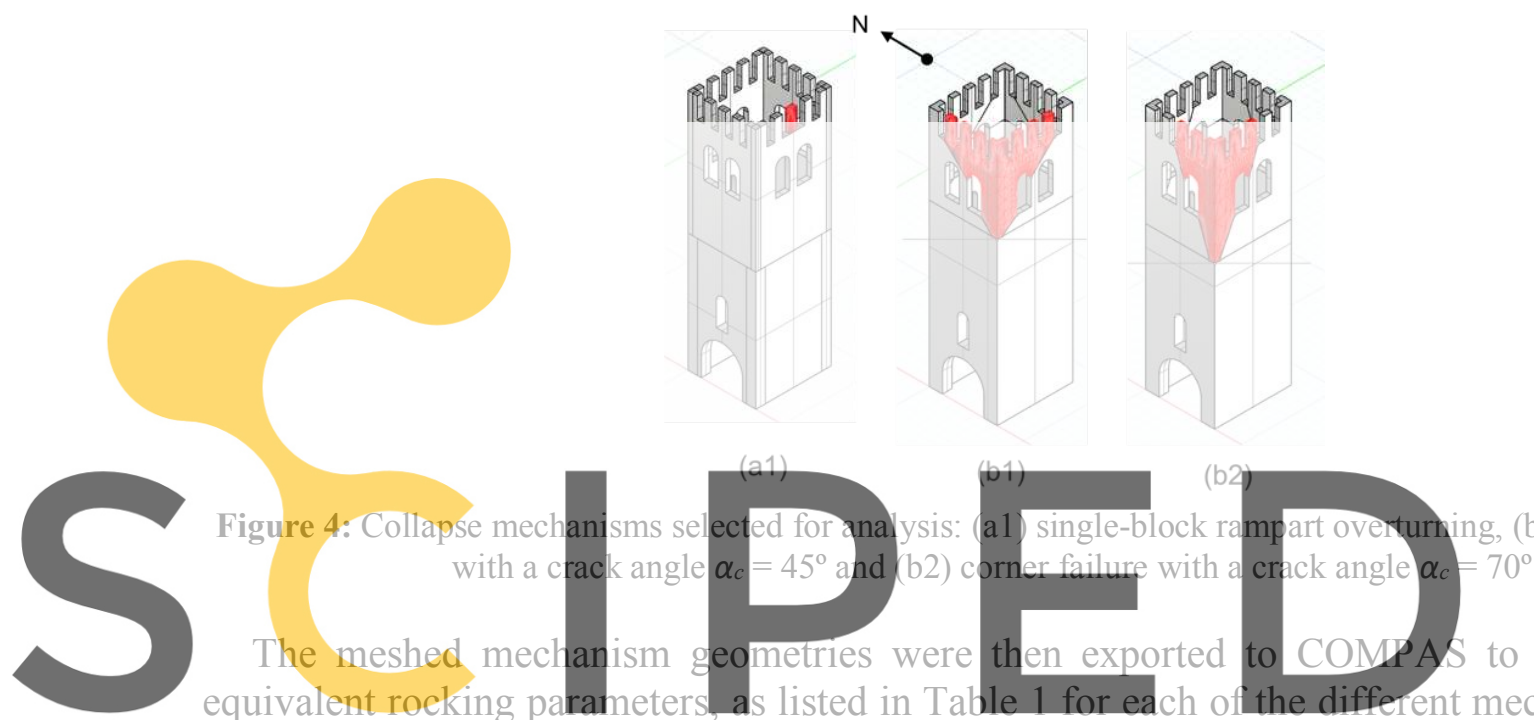
equivalent rocking parameters, as listed in Tab

that the coefficient of restitution $\eta$ is negative for Mechanisms b1 and b2 as both undergo one-

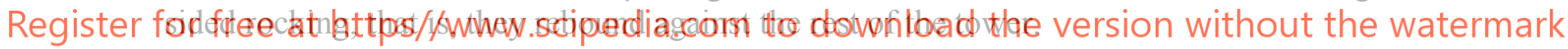

Table 1: Equivalent rocking parameters for the different mechanisms

\begin{tabular}{cccc}
\hline & a1 & b1 & b2 \\
\hline $\boldsymbol{p}_{e q}\left(\mathbf{s}^{-1}\right)$ & 2.55 & 1.22 & 1.12 \\
\hline$\lambda(\mathbf{r a d})$ & 0.22 & 0.33 & 0.20 \\
\hline$\phi_{o v}(\mathbf{r a d})$ & 0.22 & 0.33 & 0.20 \\
\hline $\boldsymbol{n} \boldsymbol{s}$ & 2 & 1 & 1 \\
\hline $\boldsymbol{\eta}$ & 0.93 & -0.35 & -0.56 \\
\hline $\boldsymbol{h}_{\boldsymbol{m}}(\mathbf{m})$ & 23.5 & 17.1 & 15.0 \\
\hline
\end{tabular}

\subsection{Modal analyses}

As all three mechanisms take place at a height above ground level $\left(h_{m}>h_{g}\right)$, modal analyses were conducted using compas_fea. Note that as the mechanisms all occur in the same tower, only one meshed model of the full structure was exported to COMPAS.

The bottom $8.55 \mathrm{~m}$ of the tower is connected to the adjacent buildings on its northern and southern façades, but limited information is available about the type/level of connectivity. 
Therefore, two different boundary conditions were considered to provide a lower and upper bound for this parameter. These are the isolated case (zero connectivity to the adjacent structures) and the fixed case (tower completely restrained, i.e. pinned, to the adjacent structures). The material properties assumed for both models were that of an elastic isotropic material characterized by a Young's modulus $E=0.84 \mathrm{GPa}$, a Poisson's ratio $v=0.2$ and density $\rho=2000 \mathrm{~kg} / \mathrm{m}^{3}$.

The results of the modal analyses were then used to define two SDF elastic oscillators, characterized by average frequencies of $1.55 \mathrm{~Hz}$ and $2.74 \mathrm{~Hz}$ for the isolated and fixed cases respectively, and first mode shapes as illustrated by Figure 2.

\subsection{Full time-history analyses}

Full time-history analyses were subsequently conducted for the three different collapse mechanisms for a suite of earthquake ground motions. Using the PEER NGA-West2 ground motion database, fifteen different ground motions were selected for this analysis, scaled to the site-specific response spectrum as defined in Eurocode 8, assuming soil type C, and as illustrated by Figure 5
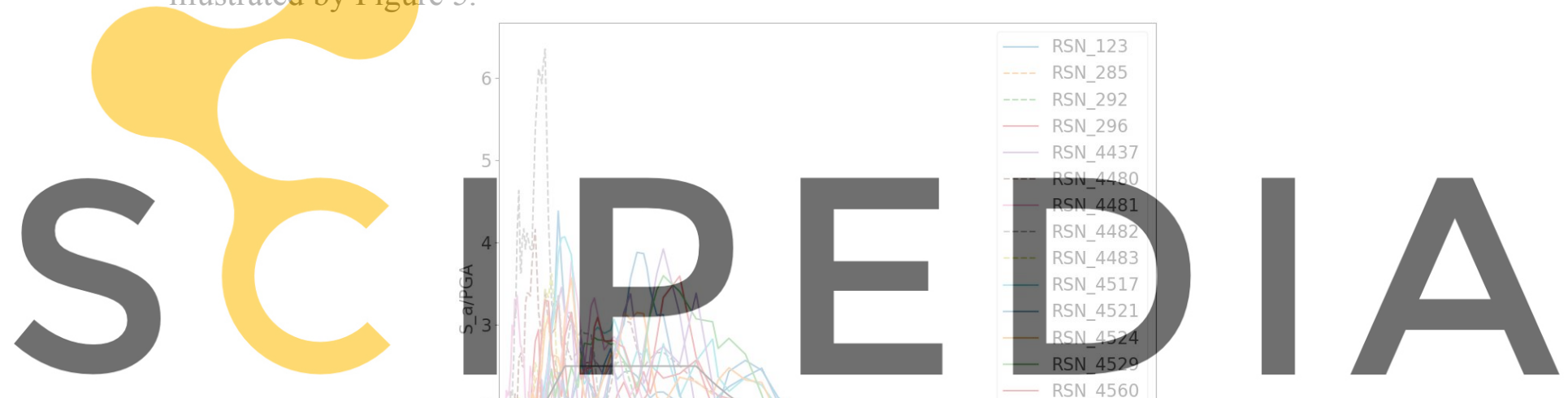

Register for free at https//www.scipedia.com to download the version without the watermark

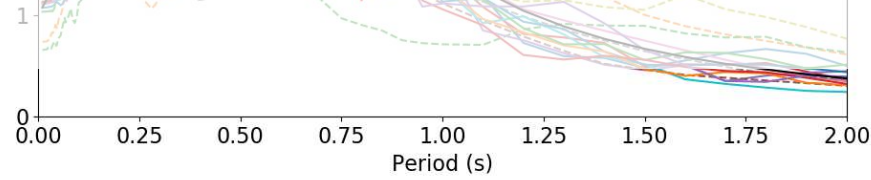

Figure 5: Elastic acceleration spectra (normalized by PGA) of the ground motions selected for analysis. Note that the site-specific target spectrum is shown in black.

Equation (5) was then solved for the two SDF elastic oscillators (for the isolated and fixed cases) for each of the ground motions, and the response scaled to get the final input signals at the base of the three rocking mechanisms. The filtered and scaled input signals were then substituted into Equation (3), which was solved to predict the response of each of the rocking mechanisms to the different ground motions, for both the isolated ("iso") and fixed cases, with the predictions expressed in terms of rotation $\phi$ over time. For each mechanism, the maximum predicted rotation $\phi_{\max }$ normalized by the overturning rotation $\phi_{o v}$, was then compared for each of the different ground motion records, for both connectivity cases ("iso" and "fixed"), as illustrated by Figure 6 for Mechanism al (scaled to a PGA $=0.1 \mathrm{~g}$ ). 
As Figure 6 illustrates, for the isolated case, the tower fails via Mechanism a1 for 4 out of 15 of the earthquake records when the ground motion is scaled to PGA $=0.1 \mathrm{~g}$. Failure in this case is defined as overturning collapse, with overturning taking place when $\phi_{\max } / \phi_{o v}=1$, although failure would occur at lower rotations in reality. For the full suite of earthquake ground motions, $\phi_{\max } / \phi_{o v}$ had a median value of 0.71 . For the fixed case, i.e. for the tower connected to the adjacent structures, the predictions of the rocking equation of motion are consistently lower than for the isolated case (with the exception of RSN 4437 where the two predictions are equal), with a median value of 0.30 for $\phi_{\max } / \phi_{o v}$ for the entire suite of ground motion records.

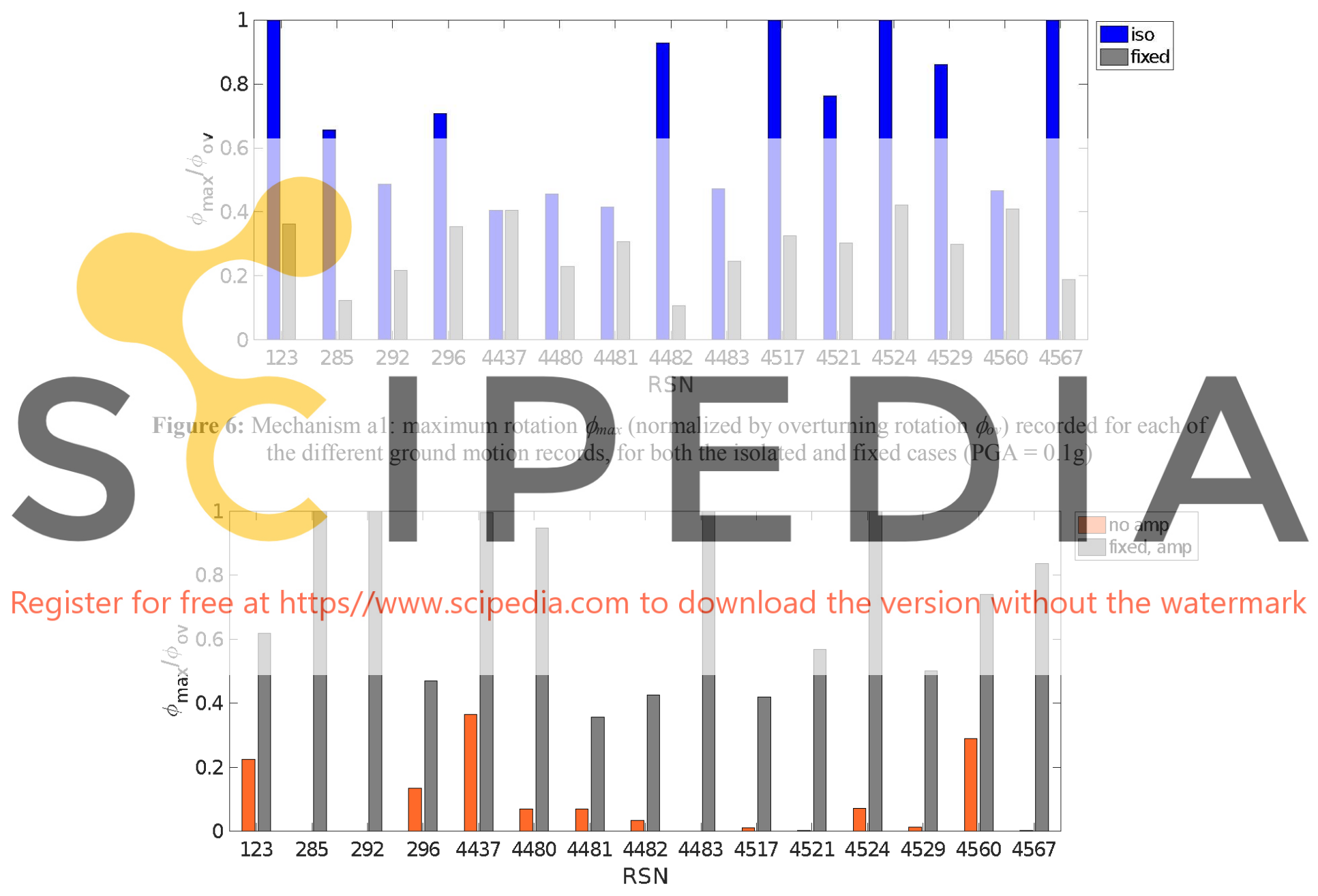

Figure 7: Mechanism a1: maximum rotation $\phi_{\max }$ (normalized by overturning rotation $\phi_{o v}$ ) recorded for each of the different ground motion records, for both the case without amplification and with amplification (fixed case) $(\mathrm{PGA}=0.25 \mathrm{~g})$

To demonstrate the importance of accounting for amplification effects, another set of analyses were conducted on Mechanism a1, now including the case where amplification effects were neglected, that is, the input ground motion $\ddot{u}_{g}$ for Equation (3) was set equal to the original ground motion $a_{g}$. For rocking to initiate in this case, the ground motion records were now scaled to a PGA of $0.25 \mathrm{~g}$ and the results of these analyses are presented in Figure 7 . Note that 
only the fixed case results are provided for comparison here, as the isolated case resulted in overturning failure for all considered ground motion records. As Figure 7 illustrates, this level of scaling of the ground motion results in collapse of the mechanism for the fixed case (i.e. with amplification) for 5 out of 15 of the selected earthquake records, with a median value of 0.74 for $\phi_{\max } / \phi_{o v}$ for the entire suite. Neglecting amplification effects ("no amp") on the other hand results in comparatively smaller rotations being predicted for the structure, with a median value of 0.03 for $\phi_{\max } / \phi_{o v}$ for the entire suite.

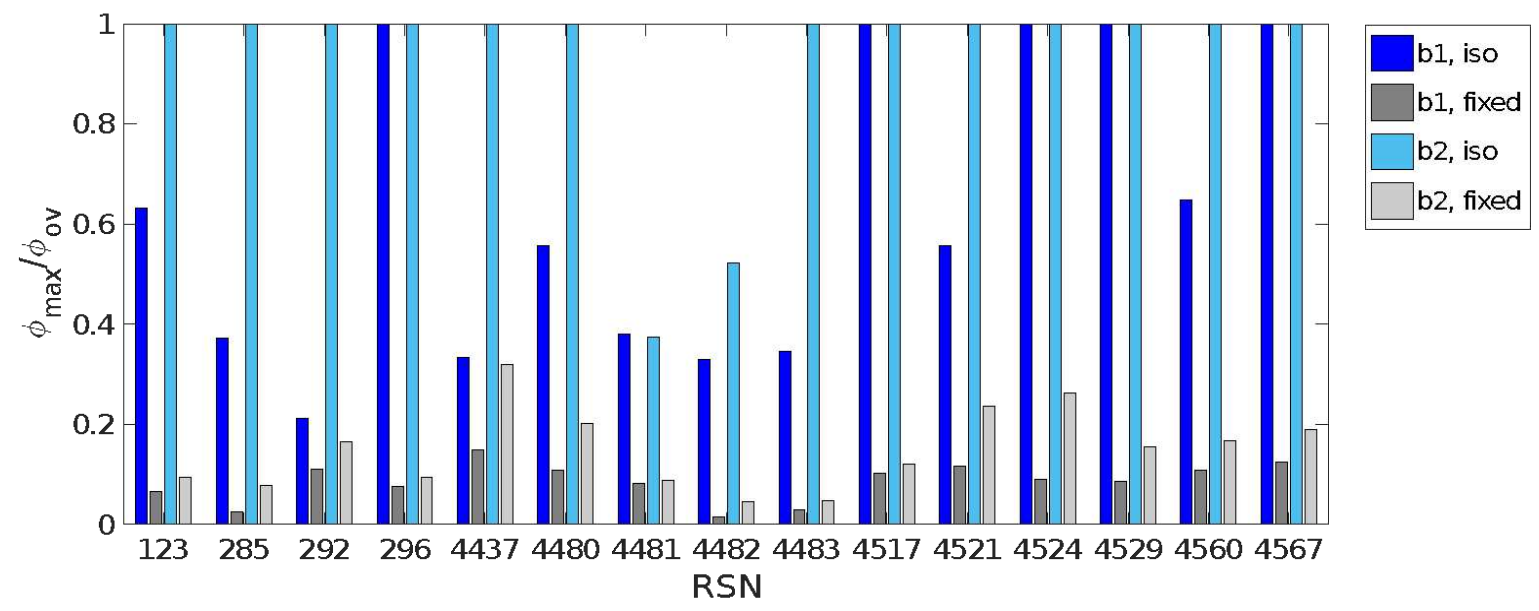

Figure 8: Mechanisms b1 and b2: maximum rotations $\phi_{\max }$ (normalized by overturning rotation $\phi_{o v}$ ) recorded for each of the different ground motion records, for both the isolated and fixed cases $(\mathrm{PGA}=0.5 \mathrm{~g})$

The final set of time-history analyses were conducted on Mechanisms b1 and b2, with the ground motion now scaled to a PGA $=0.5 \mathrm{~g}$ to initiate rocking for the relatively stockier Mechanism b1. The results of these analyses are presented in Figure 8 for both the isolated and fixed cases. As Figure 8 illustrates, this level of ground motion scaling results in failure via Mechanism b1 for 5 out of 15 and failure via Mechanism b2 for 13 out of 15 of the earthquake records for the isolated case, with median $\phi_{\max } / \phi_{o v}$ values of 0.56 and 1.00 respectively. It is clear that in this case the slenderness of $\mathrm{b} 2(\lambda=0.20$, as opposed to $\lambda=0.33$ for $b 1)$ controls the response, resulting in the overturning of b2 more frequently than $\mathrm{b} 1$ - despite the fact that both mechanisms are of similar scale, with b1 also occurring slightly higher up than b2 and consequently experiencing a greater degree of amplification. Similarly, for the fixed case, Mechanism b1 records smaller median $\phi_{\max } / \phi_{o v}$ values than Mechanism b2 (0.09 and 0.16 respectively). As in the case of Mechanism a1, the predictions for the fixed case are also considerably less conservative than their isolated counterparts.

\section{CONCLUSIONS}

In this paper, a new modeling approach for the seismic collapse assessment of masonry structures is presented, which integrates finite element analysis with rocking dynamics to model the dynamic response of complex structural geometries in a computationally efficient manner. This new modeling strategy, implemented using the COMPAS framework, is first described, following which it is applied to the seismic assessment of a historic masonry tower in NorthEastern Italy. 
For the level of seismic hazard expected on site, failure of the tower is most likely to occur via overturning of one of the merlon/rampart elements. The results also illustrated the importance of accounting for ground motion amplification effects, as well as the influence of varying boundary conditions (isolated vs fixed) on the dynamic response.

\section{REFERENCES}

[1] D'Ayala, D. and Speranza, E. An Integrated Procedure for the Assessment of Seismic Vulnerability of Historic Buildings. In: Proceedings of the 12th European Conference on Earthquake Engineering, (2002).

[2] PCM-DPC MiBAC. Model A-DC Scheda per il rilievo del danno ai beni culturali-Chiese. (2006).

[3] Housner, G.W. The Behavior of Inverted Pendulum Structures during Earthquakes. Bull. Seismol. Soc. Am. (1963) 53: 403-417.

[4] Mauro, A., de Felice, G. and DeJong, M.J. The relative dynamic resilience of masonry collapse mechanisms. Eng. Struct. (2015) 85:182-194.

[5] DeJong, M.J. and Dimitrakopoulos, E.G. Dynamically equivalent rocking structures. Earthq. Eng. Struct. Dyn. (2014) 43:1543-1563.

[6] Makris, N. and Vassiliou, M.F. Planar rocking response and stability analysis of an array of free-standing columns capped with a freely supported rigid beam. Earthq. Eng. Struct. Dyn. (2013) 42:431-449.

[7] Priestley, M.J.N. Seismic behaviour of unreinforced masonry walls. Bull. New Zeal. Soc. Earthq. Eng. (1985) 18:191-205.

[8] Casarin, F. and Modena, C. Seismic Assessment of Complex Historical Buildings: Application to Reggio Emilia Cathedral, Italy. Int. J. Archit. Herit. (2008) 2:304-327.

[9] Castellazzi, G., Gentilini, C. and Nobile, L. Seismic Vulnerability Assessment of a Historical Church: Limit Analysis and Nonlinear Finite Element Analysis. Adv. Civ. Eng. (2013) 2013:1-12.

[10] de Felice, G., Genoese, A., Genoese, A. and Malena, M. Seismic Vulnerability Assessment of the Casamari Gothic Church. In: Proceedings of the 15th International Conference on Civil, Structural and Environmental Engineering Computing, (2015).

[11] Van Mele, T. et al. COMPAS: A framework for computational research in architecture and structures. (2017-2020).

[12] Mehrotra, A. and DeJong, M.J. A CAD-interfaced dynamics-based tool for analysis of masonry collapse mechanisms. Eng. Struct. (2018) 172: 833-849.

[13] Liew, A. and Mendez Echenagucia, T. compas_fea: Finite Element Analysis package for the COMPAS framework. (2017).

[14] McKenna, F., Fenves, G.L., Scott, M.H. and Jeremic, B. Open system for earthquake engineering simulation (OpenSees). (2000).

[15] Robert McNeel \& Associates. Rhinoceros 5. (2014).

[16] Klöckner, A. MeshPy. (2008).

[17] Si, H. TetGen, a Delaunay-Based Quality Tetrahedral Mesh Generator. ACM Trans. Math. Softw. (2015) 41: Article 11.

[18] Valente, M. and Milani, G. Seismic assessment of historical masonry towers by means of simplified approaches and standard FEM. Constr. Build. Mater. (2016) 108:74-104. 